\title{
Antibacterial Properties of Methanolic and Aqueous Extracts of Some Plants against Some Enterobacteriaceae Species
}

\author{
Sana Eltayeb Mahjoob Hamed1, Hatil Hashim EL-Kamali²* \\ ${ }^{1}$ Department of Biochemistry, Faculty of Medical Laboratories Sciences, Omdurman Islamic University, \\ Omdurman, Sudan \\ ${ }^{2}$ Department of Botany, Faculty of Science and Technology, Omdurman Islamic University, Omdurman, Sudan \\ Email: "hatilhashim@gmail.com
}

Received 3 March 2016; accepted 18 March 2016; published 22 March 2016

Copyright (C) 2016 by authors and OALib.

This work is licensed under the Creative Commons Attribution International License (CC BY). http://creativecommons.org/licenses/by/4.0/

(c) (i) Open Access

\section{Abstract}

The extracts of six medicinal plants namely Terminalia chebula fruits, Commiphora myrrha gum, Solenostemma argel leaves, Rutagraveolens aerial parts, Cistanche phelypaea aerial parts and Striga hermonthica stem used in traditional Sudanese medicine for the treatment of gastrointestinal tract infections were selected to evaluate their potential antibacterial activity. The antibacterial activity of methanolic and aqueous extracts of these plants were determined by agar diffusion technique in vitro against 20 clinical isolates ( 2 were Salmonella typhi, 5 Proteus mirabilis, 4 Escherichia coli, 5 Pseudomonas aeruginosa, 3 Staphylococcus aureus, one was Salmonella paratyphi B) and 5 standard bacterial strains (Staphylococcus aureus ATCC 25923), Bacillus subtilis (NCTC 8236), Escherichia coli (ATCC 25922), Salmonella typhi (ATCC1319106) and Klebsiella pneumoniae (ATCC 35657) at a concentration of $100 \mathrm{mg} / \mathrm{ml}$. Of all plants methanolic and aqueous extracts of $T$. chebula fruits were the most active with clinical isolates and standard bacterial strains showed relatively high antibacterial activity against most of the tested microorganisms with the diameter of inhibition zones ranging between 20 and $24 \mathrm{~mm}$, whereas the methanolic extract of Commiphora myrrha showed high antibacterial activity against Proteus mirabilis and Escherichia coli clinical isolate $(1 \mathrm{Z}=20 \mathrm{~mm})$. Solenostemma argel leaves was found moderately effective against $S$. aureus (ATCC $25923((1 \mathrm{Z}=18 \mathrm{~mm})$ but did not show any activity against all tested clinical isolates bacteria. Most susceptible Gram-negative clinical Isolates bacteria were Escherichia coli and Proteus mirabilis. Most susceptible Gram negative standard bacteria were Bacillus subtilis (NCTC 8236) and Escherichia coli (ATCC 25922) and least susceptible Gram negative bacterium was Klebsiella pneumoniae (ATCC35657). In Gram positive standard bacteria, most susceptible was $S$. aureus (ATCC 25923). Antibiotics was used as standards drug for antibacterial assay. The present study reveals potential use of these plants for developing new antibacterial compounds against gastrointestinal tract pathogenic microorganisms.

${ }^{*}$ Corresponding author.

How to cite this paper: Hamed, S.E.M. and EL-Kamali, H.H. (2016) Antibacterial Properties of Methanolic and Aqueous Extracts of Some Plants against Some Enterobacteriaceae Species. Open Access Library Journal, 3: e2488.

http://dx.doi.org/10.4236/oalib.1102488 


\title{
Keywords
}

\section{Antibacterial Activity, Medicinal Plants, Clinical Isolates and Standard Pathogenic Bacteria}

\author{
Subject Areas: Biochemistry
}

\section{Introduction}

The use of medicinal plants to treat human diseases has its roots in pre-historical times. Despite of the modern advances achieved in the field of synthetic chemistry, the most efficient drugs available had derived directly or indirectly, related from plant kingdom. Indigenous communities had long used plant extracts to treat illness. Many of these extracts had shown effective action with new bioactive compounds being extracted and screened every year. These extracts had also proven to be good sources of therapeutic agents to the treatment of infectious diseases [1] [2]. Sudan throughout its long history has accumulated a rich body of empirical knowledge of the use of medicinal plants for the treatment of various diseases.

The antimicrobial activity of Terminalia chebula against a variety of bacterial and fungal strains has been well investigated by several researchers [3]-[20].

The antibacterial and fungal activities of Solenostemma argel [21] [22], Comiphora myrrha [24]-[27] and Cistanche phelypaea [28] have been received considerable.

The main aim of this study is that due to increasing concerns about the development of antimicrobial resistance among pathogenic bacteria, so alternative strategies are sought that do not use antibiotics to reduce pathogenic bacteria from foods and patients.

\section{Materials and Methods}

The fruits of Terminalia chebula, Commiphora myrrha gum, Cistanche phelypaea root, Striga hermonthica stem, and Solenostemma argel leaves were purchased from Omdurman market on the basis of undocumented reports for antibacterial activity. The plants were identified by one author Prof. Hatil H. Elkamali and by comparison with herbarium of the Department of the Botany Department, Faculty of Science and Technology, Omdurman Islamic University. The dried plants were pulverized with a mechanical grinder.

Two hundred gramsofall plants was macerated separately with $50 \%$ methanol $(\mathrm{MeOH})$ in a conical flask for 24 hours. Mother liquor (crude $\mathrm{MeOH}$ extract) was filtered and evaporated to dryness. The dry crude extract was sterilized. All extracts were stored dry in sterilized containers at room temperature until used for antibacterial testing. At the time of testing, the extracts were prepared at a concentration of $100 \mathrm{mg} / \mathrm{ml}$ in methanol.

Dried plant material $(100 \mathrm{~g})$ was ground to a fine powder. It was macerated with distilled water $(1 \mathrm{~L})$, and left for 24 hours at room temperature. The mother liquor was filtered. The filtrate, thus obtained was evaporated to complete dryness at room temperature. The residue thus obtained was aqueous plant extract.

The antibacterial activity was tested by well-agar diffusion method [29] [30]. $250 \mathrm{ml}$ of sterilized nutrient agar was used for testing. The inoculum size of each test organism was adjusted to suspension of $10^{6}$ cells. $2 \mathrm{ml}$ of 24 hours old culture of bacteria were added to $250 \mathrm{ml}$ of melted cooled test agar and after through mixing, approximately $20 \mathrm{ml}$ of this seeded agar were poured into $10 \mathrm{~cm}$ diameter presterilized petri dishes and allowed to solidify. Four wells (10 mm in diameter) were bored in the agar using a sterile cork borer and the agar discs were removed. $0.1 \mathrm{ml}$ aliquots of the prepared extract was placed into a well with a pipette and the plate was held for 2 hours at room temperature for diffusion of extract into agar. Subsequently, the plate was incubated at $37^{\circ} \mathrm{C}$ for 24 hours. After incubation, the diameter of the zones of inhibition were measured to the nearest mm.

The minimum inhibitory concentration (MIC) was determined by a modification of the agar diffusion method [29]. A two-fold serial dilution of each extract was prepared in methanol to achieve a decreasing range of extract concentrations from $100 \mathrm{mg} / \mathrm{ml}$ to approximately $3.125 \mathrm{mg} / \mathrm{ml}$. A $0.1 \mathrm{ml}$ sample of each dilution was introduced into duplicate wells in a nutrient agar plate already seeded with bacterial cells as described above. Incubation was at $37^{\circ} \mathrm{C}$ for 24 hours. The lowest concentration of extract showing a zone of inhibition was taken as the MIC.

Multidisc for antimicrobial susceptibility testing from Axiom laboratories, New Delhi, India for were used as 
positive control and methanol as a negative control.

\section{Results and Discussion}

Some bacterial strains showed a fairly high degree of sensitivity to the methanolic extracts of $T$. chebula fruits such as $P$. mirabilis isolates no. $(3,4), E$. coli isolates no. $(5,10), P$. aeruginosa $(7,8,13)$ and $S$. aureus isolate no. (12) $(1 \mathrm{Z}=$ range between $20-28 \mathrm{~mm})$. Standard bacteria, S. aureus (ATCC 25923) and B. subtilis (NCTC 8236) showed promising result ( $1 \mathrm{Z}$ = range between 18 - $24 \mathrm{~mm}$ ), bacterial species: S.typhi (ATCC1319106), $K$. pneumoniae (ATCC 35657) and E. coli (ATCC 25922) were found to be resistant (Table 1). Methanol extracts of Commiphora myrrha gum showed high antibacterial activity against $P$. mirabilis isolate no. (3) and E. coli isolate no. (6) $(1 \mathrm{Z}=20 \mathrm{~mm})$. Solenostemma argel leaves was found effective against $S$. aureus (ATCC 25923 $((1 \mathrm{Z}=18 \mathrm{~mm})$ (Table 1). Methanol extract of R.graveolens aerial parts, Cistanche phelypaea roots, Striga hermonthica stem, were found ineffective against all tested Gram-positive and Gram-negative bacteria.

All bacterial species were found to be resistant against aqueous extracts of all plants except $T$. chebula fruits (Table 1). Aqueous extract of Terminalia chebula fruits was found moderately active to $S$. typhi $B$ isolate no. (17), S. typhi isolate no. (1), E.coli isolate no. (6), P. aeruginosa isolate no. (8) and P.mirabilis isolate no. (19) $(1 \mathrm{Z}$ = range between $16-14 \mathrm{~mm}$ ) (Table 1). B. subtilis (NCTC 8236) and S. aureus (ATCC 25923) showed moderate result $(1 \mathrm{Z}=$ range between $16-14 \mathrm{~mm})$. Bacterial species: E. coli (ATCC 25922), S. typhi (ATCC1319106), K. pneumonia (ATCC 35657) were found to be resistant (Table 1).

Solenostemma argel leaves was found effective against S.aureus (ATCC 25923) $(1 \mathrm{Z}=18 \mathrm{~mm}$ ), but did not showed any activity against all tested clinical isolates bacteria (Table 1). Methanolic and aqueous extracts of R.graveolens aerial parts, Cistanche phelypaea roots and Striga hermonthica stem, were found ineffective against all tested bacteria (Table 1).

The antibacterial activity related to known antibiotics was calculated. The results are shown in Table 2.

Ceftizoxime was found effective against $P$. mirabilis isolate no. (2) and $P$. aeruginosa isolate no. (13) at concentration $30 \mathrm{mcg}$, and moderate effectively was observed against $P$. aeruginosa isolate no. (15) and $E$. coli (ATCC 25922). Cefotaxime (CF) showed promising result against $P$. mirabilis isolate no. (4) at concentration $30 \mathrm{mcg}$ (Table 2). It was showed no antibacterial activity against S. aureus (ATCC 25923) and S. typhi (ATCC1319106). Ciprofloxacin (CP) showed a fairly high degree of sensitivity to E. coli isolate no. (6), P. aeruginosa isolate no. (7), S. typhi B isolate no. (7) and B. subtilis (NCTC 8236) at concentration $5 \mathrm{mcg}$, and demonstrated antibacterial activity against E.coli (ATCC 25922), K. pneumoniae (ATCC 35657) (Table 3).

Tetracycline (TE) showed good results against $E$. coli isolate no. (6) and E. coli (ATCC 25922) at concentration $30 \mathrm{mcg}$. Amikacin (AK) showed high antibacterial activity against E. coli isolate no. (10) at concentration $30 \mathrm{mcg}$, and moderate effectively was observed against $E$. coli isolate no. (5), P. aeruginosa isolate no. (7) and E. coli (ATCC 25922). It was showed no antibacterial activity against $S$. aureus (ATCC 25923) and S. typhi (ATCC1319106). S. aureus (ATCC 25923), B. subtilis (NCTC 8236), E. coli (ATCC 25922), S. typhi (ATCC1319106) and K. pneumoniae (ATCC 35657) was found to be co-trimoxazole resistant. Co-Trimoxazole was found effective against $S$. typhi $B$ isolate no. (17) at concentration $25 \mathrm{mcg}$ and showed good results against $E$. coli isolate no. (9), S. aureus (14) and $P$. aeruginosa isolate no. (15).

Piperacillin/Tazobactam (TZP) showed good results against E. coli isolate no. (6) at concentration 100/10 mcg, and found to be ineffective against all standard bacteria except B. subtilis (NCTC 8236). Chloramphenicol $(\mathrm{CH})$ showed promising result against $B$. subtilis (NCTC 8236) at concentration $30 \mathrm{mcg}$, and moderate effectively was observed against E. coli isolate no. (9) S. aureus isolate no. (14), P. aeruginosa isolate no. (15) and $P$. mirabilis isolate no. (19), whereas S. aureus (ATCC 25923), E. coli (ATCC 25922), S. typhi (ATCC1319106) and K. pneumoniae (ATCC 35657) was found to be chloramphenicol resistant.

Methanolic extracts of $T$. chebula fruits showed high antibacterial activity against clinical isolates and moderate to some of standard bacteria, about $60 \%$ of $P$. mirabilis revealed promising sensitivity, $50 \%$ of $S$. aureus clinical isolates showed good activity and surprise sensitivity of standard S. aureus (ATCC 25923) has become resistant to all known antibiotics has posed a threat already for a number of years. It has thus become apparent that new antimicrobial agents will continue to select for resistant strains from the pool of bacteria which continuously undergo genetic change [31]. T. chebula can serve as a starting point in future drug development aimed at the production of a new safe, effective and bio-accessible therapeutic agent.

The polar extracts of $T$. chebula fruits, (methanol) exhibited promising antibacterial activity against most Pro- 
Table 1. Antibacterial activity of methanolic and aqueous extracts of studied plants against clinical isolates and standard bacteria.

\begin{tabular}{|c|c|c|c|c|c|c|c|c|}
\hline ż & 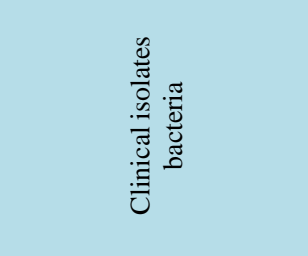 & 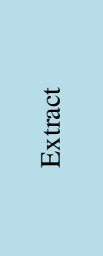 & 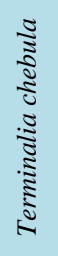 & 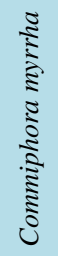 & 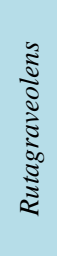 & 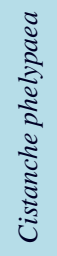 & 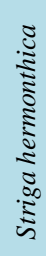 & 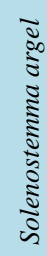 \\
\hline \multirow{2}{*}{1} & \multirow{2}{*}{ Salmonella typhi } & $\mathrm{MeOH}$ & 16 & 2 & - & - & - & 2 \\
\hline & & $\mathrm{H}_{2} \mathrm{O}$ & 14 & - & - & - & - & 4 \\
\hline \multirow{2}{*}{2} & \multirow{2}{*}{ Proteus mirabilis } & $\mathrm{MeOH}$ & 18 & 2 & 2 & - & 2 & 2 \\
\hline & & $\mathrm{H}_{2} \mathrm{O}$ & 8 & - & - & - & - & 6 \\
\hline \multirow{2}{*}{3} & \multirow{2}{*}{ Proteus mirabilis } & $\mathrm{MeOH}$ & 20 & 20 & 12 & 2 & 2 & 2 \\
\hline & & $\mathrm{H}_{2} \mathrm{O}$ & 8 & 6 & - & 8 & - & 2 \\
\hline \multirow{2}{*}{4} & \multirow{2}{*}{ Proteus mirabilis } & $\mathrm{MeOH}$ & 28 & 2 & 2 & 2 & 2 & 4 \\
\hline & & $\mathrm{H}_{2} \mathrm{O}$ & 4 & - & - & - & - & 4 \\
\hline \multirow{2}{*}{5} & \multirow{2}{*}{ Escherichia coli } & $\mathrm{MeOH}$ & 24 & 2 & - & - & 2 & 2 \\
\hline & & $\mathrm{H}_{2} \mathrm{O}$ & 10 & - & - & - & - & 6 \\
\hline \multirow{2}{*}{6} & \multirow{2}{*}{ Escherichia coli } & $\mathrm{MeOH}$ & 10 & 20 & - & 2 & 4 & - \\
\hline & & $\mathrm{H}_{2} \mathrm{O}$ & 14 & 6 & - & 6 & - & 4 \\
\hline \multirow{2}{*}{7} & \multirow{2}{*}{ Pseudomonas aeruginosa } & $\mathrm{MeOH}$ & 20 & - & - & 2 & - & 2 \\
\hline & & $\mathrm{H}_{2} \mathrm{O}$ & 6 & - & - & 4 & - & 10 \\
\hline \multirow{2}{*}{8} & \multirow{2}{*}{ Pseudomonas aeruginosa } & $\mathrm{MeOH}$ & 20 & 4 & 2 & 2 & 4 & - \\
\hline & & $\mathrm{H}_{2} \mathrm{O}$ & 14 & - & - & 4 & - & 6 \\
\hline \multirow{2}{*}{9} & \multirow{2}{*}{ Escherichia coli } & $\mathrm{MeOH}$ & 2 & 2 & 2 & 2 & 2 & - \\
\hline & & $\mathrm{H}_{2} \mathrm{O}$ & 4 & - & - & 6 & - & 4 \\
\hline \multirow{2}{*}{10} & Escherichia coli & $\mathrm{MeOH}$ & 28 & - & - & 2 & 2 & - \\
\hline & Escherichia coli & $\mathrm{H}_{2} \mathrm{O}$ & 10 & - & - & 4 & - & - \\
\hline & & $\mathrm{MeOH}$ & 4 & 12 & 6 & 2 & - & - \\
\hline 11 & Staphylococcus aureus & $\mathrm{H}_{2} \mathrm{O}$ & 8 & 6 & - & 10 & - & 6 \\
\hline & & $\mathrm{MeOH}$ & 20 & 2 & - & - & 2 & - \\
\hline 12 & Staphylococcus aureus & $\mathrm{H}_{2} \mathrm{O}$ & 4 & - & - & - & - & 4 \\
\hline & & $\mathrm{MeOH}$ & 20 & 6 & 6 & 4 & 6 & 4 \\
\hline 13 & Pseudomonas aeruginosa & $\mathrm{H}_{2} \mathrm{O}$ & - & 2 & - & - & - & - \\
\hline & & $\mathrm{MeOH}$ & 2 & 2 & 6 & - & 2 & - \\
\hline 14 & Staphylococcus aureus & $\mathrm{H}_{2} \mathrm{O}$ & 4 & 6 & - & - & - & 4 \\
\hline & & $\mathrm{MeOH}$ & 10 & - & 2 & - & 2 & - \\
\hline 15 & Pseudomonas aeruginosa & $\mathrm{H}_{2} \mathrm{O}$ & 12 & - & - & - & - & - \\
\hline & & $\mathrm{MeOH}$ & 2 & - & 2 & - & - & - \\
\hline 16 & Proteus mirabilis & $\mathrm{H}_{2} \mathrm{O}$ & 12 & 6 & 4 & - & - & - \\
\hline & & $\mathrm{MeOH}$ & 2 & 2 & 11 & 2 & 2 & - \\
\hline 17 & Salmonella paratyphi B & $\mathrm{H}_{2} \mathrm{O}$ & 16 & 6 & - & 8 & - & 4 \\
\hline & & $\mathrm{MeOH}$ & 10 & 2 & - & - & 2 & - \\
\hline 18 & Salmonella typhi & $\mathrm{H}_{2} \mathrm{O}$ & 12 & 6 & - & - & - & 2 \\
\hline & & $\mathrm{MeOH}$ & 2 & - & - & 2 & 2 & 2 \\
\hline 19 & Proteus mirabilis & $\mathrm{H}_{2} \mathrm{O}$ & 14 & 6 & - & - & - & 6 \\
\hline 20 & & $\mathrm{MeOH}$ & - & - & - & - & 2 & - \\
\hline 20 & Pseudomonas aeruginosa & $\mathrm{H}_{2} \mathrm{O}$ & 12 & 6 & - & 2 & - & 8 \\
\hline & & & Star & eria & & & & \\
\hline 1 & Staphylococcus aureus & $\mathrm{MeOH}$ & 24 & - & 8 & 10 & 8 & 18 \\
\hline 1 & (ATCC 25923) & $\mathrm{H}_{2} \mathrm{O}$ & 14 & - & - & - & - & 6 \\
\hline 2 & Bacillus subtilis & $\mathrm{MeOH}$ & 18 & 8 & 10 & 8 & 8 & 4 \\
\hline & (NCTC 8236) & $\mathrm{H}_{2} \mathrm{O}$ & 16 & 6 & - & - & - & - \\
\hline & Escherichia coli & $\mathrm{MeOH}$ & 6 & 6 & 8 & 2 & 4 & 4 \\
\hline 3 & (ATCC 25922) & $\mathrm{H}_{2} \mathrm{O}$ & 6 & - & - & - & - & - \\
\hline & Klebsiella pneumonia & $\mathrm{MeOH}$ & 4 & 2 & 2 & 2 & 2 & 2 \\
\hline 4 & (ATCC 35657) & $\mathrm{H}_{2} \mathrm{O}$ & 10 & - & - & - & - & 4 \\
\hline & Salmonella typhi & $\mathrm{MeOH}$ & 12 & 2 & 4 & 2 & 2 & 2 \\
\hline 5 & (ATCC1319106) & $\mathrm{H}_{2} \mathrm{O}$ & 10 & 6 & - & - & - & 2 \\
\hline
\end{tabular}

Values are the mean of four replicates; -: no inhibition. Tested concentration of extracts: $100 \mathrm{mg} / \mathrm{ml}(0.1 \mathrm{ml} /$ well). Methanol did not show any inhibitory activity. Sensitive: >18, intermediate: 14 - $18 \mathrm{~mm}$, Resistant: <14 mm, -: No inhibition zone. ATCC: American Type Collection Culture-NCTC: National Collection Type Culture. 
Table 2. Antibacterial activity of antibiotics-Gram (-ve) against clinical isolates.

\begin{tabular}{|c|c|c|c|c|c|c|c|c|c|c|c|c|c|}
\hline & $\begin{array}{l}\text { Antibiotics } \\
\text { Clinical isolates }\end{array}$ & $\begin{array}{c}\text { AS } \\
20 \mathrm{mcg}\end{array}$ & $\begin{array}{c}\text { BA } \\
25 \mathrm{mcg}\end{array}$ & $\begin{array}{c}\mathrm{CF} \\
30 \mathrm{mcg}\end{array}$ & $\begin{array}{c}\text { TZP } \\
100 / 10 \\
\text { mcg }\end{array}$ & $\begin{array}{c}\mathrm{CH} \\
30 \mathrm{mcg}\end{array}$ & $\begin{array}{c}\mathrm{CP} \\
5 \mathrm{mcg}\end{array}$ & $\begin{array}{c}\text { CI } \\
30 \mathrm{mcg}\end{array}$ & $\begin{array}{c}\mathrm{TE} \\
30 \mathrm{mcg}\end{array}$ & $\begin{array}{c}\text { OF } \\
5 \mathrm{mcg}\end{array}$ & $\begin{array}{c}\text { GM } \\
10 \mathrm{mcg}\end{array}$ & $\begin{array}{c}\text { AK } \\
30 \mathrm{mcg}\end{array}$ & $\begin{array}{c}\text { GF } \\
5 \mathrm{mcg}\end{array}$ \\
\hline 1 & Salmonella typhi & 2 & - & 14 & 10 & 8 & 10 & 14 & 2 & 10 & 10 & 10 & 10 \\
\hline 2 & Proteus mirabilis & 8 & 10 & 2 & 12 & 8 & 10 & 20 & 2 & 10 & 10 & 10 & 10 \\
\hline 3 & Proteus mirabilis & - & - & - & - & - & - & - & - & - & - & - & - \\
\hline 4 & Proteus mirabilis & - & - & 20 & - & - & 10 & - & - & - & - & - & - \\
\hline 5 & Escherichia coli & 6 & - & 12 & 12 & 10 & - & - & 2 & 8 & 14 & 14 & 14 \\
\hline 6 & Escherichia coli & - & 6 & 8 & 16 & - & 20 & - & 20 & 10 & 12 & 10 & 8 \\
\hline 7 & Pseudomonas aeruginosa & - & 10 & 4 & 12 & - & 24 & - & 4 & 18 & 10 & 14 & 18 \\
\hline 8 & Pseudomonas aeruginosa & - & - & - & - & - & - & - & - & - & - & - & - \\
\hline 9 & Escherichia coli & 4 & 16 & 6 & 12 & 14 & 6 & 12 & - & 6 & - & - & 12 \\
\hline 10 & Escherichia coli & - & - & - & 12 & 8 & 14 & - & 10 & 14 & 18 & 20 & 14 \\
\hline 11 & Staphylococcus aureus & - & - & - & - & - & - & - & - & - & - & - & - \\
\hline 12 & Staphylococcus aureus & 2 & - & - & 6 & - & - & - & - & 6 & - & - & 16 \\
\hline 13 & Pseudomonas aeruginosa & 10 & 10 & 10 & 10 & 10 & 12 & 20 & 6 & 10 & 8 & 8 & 10 \\
\hline 14 & Staphylococcus aureus & - & 14 & 2 & - & 16 & 8 & 12 & - & 4 & - & - & 10 \\
\hline 15 & Pseudomonas aeruginosa & - & 14 & 4 & - & 16 & 10 & 16 & - & 6 & 16 & 8 & 14 \\
\hline 16 & Proteus mirabilis & - & - & - & - & - & - & - & - & - & - & - & - \\
\hline 17 & 7 Salmonella paratyphi B & 6 & 26 & 8 & 12 & 4 & 30 & - & 12 & 18 & 14 & 8 & 16 \\
\hline 18 & Salmonella typhi & - & - & - & - & - & - & 6 & - & - & 10 & 6 & - \\
\hline 19 & Proteus mirabilis & 2 & 12 & 2 & 12 & 16 & 4 & 10 & - & 4 & - & - & 10 \\
\hline 20 & Pseudomonas aeruginosa & - & - & - & - & - & - & - & - & - & - & - & - \\
\hline
\end{tabular}

Values are the mean of four replicates; -: no inhibition. Tested concentration of extracts: $100 \mathrm{mg} / \mathrm{ml}(0.1 \mathrm{ml} /$ well); Methanol did not show any inhibitory activity. Sensitive: >18, intermediate: 14 - $18 \mathrm{~mm}$, Resistant: <14 mm, -: No inhibition zone; Multidisk for Antimicrobial Susceptibility Testing For Gram Negative Isolates: -Ampicillin/sulbactam AS 20 mcg, Co-Trimoxazole BA 25 mcg, Cefotaxime CF 30 mcg; Piperacillin/Tazobactam TZP 100/10 mcg, Chloramphenicol CH 30 mcg, Ciprofloxacin CP 5 mcg, Ceftizoxime CI 30 mcg, Tetracycline TE 30 mcg, Ofloxacin OF 5 mcg, Gentamicin GM 10 mcg, Amikacin AK 30 mcg, Gatifloxacin GF 5 mcg.

Table 3. Antibacterial activity of antibiotics-Gram (-ve) against standard bacteria.

\begin{tabular}{|c|c|c|c|c|c|c|c|c|c|c|c|c|}
\hline $\begin{array}{c}\text { Antibiotics } \\
\text { Standard bacteria }\end{array}$ & $\begin{array}{c}\text { AS } \\
20 \mathrm{mcg}\end{array}$ & $\begin{array}{c}\text { BA } \\
25 \mathrm{mcg}\end{array}$ & $\begin{array}{c}\mathrm{CF} \\
30 \mathrm{mcg}\end{array}$ & $\begin{array}{c}\text { TZP } \\
100 / 10 \\
\text { mcg }\end{array}$ & $\begin{array}{c}\mathrm{CH} \\
30 \mathrm{mcg}\end{array}$ & $\begin{array}{c}\mathrm{CP} \\
5 \mathrm{mcg}\end{array}$ & $\begin{array}{c}\text { CI } \\
30 \mathrm{mcg}\end{array}$ & $\begin{array}{c}\mathrm{TE} \\
30 \mathrm{mcg}\end{array}$ & $\begin{array}{c}\text { OF } \\
5 \mathrm{mcg}\end{array}$ & $\begin{array}{c}\text { GM } \\
10 \mathrm{mcg}\end{array}$ & $\begin{array}{c}\text { AK } \\
30 \mathrm{mcg}\end{array}$ & $\begin{array}{c}\mathrm{GF} \\
5 \mathrm{mcg}\end{array}$ \\
\hline $\begin{array}{l}\text { Salmonella typhi } \\
\text { (ATCC1319106) }\end{array}$ & 4 & - & - & - & 6 & - & - & 10 & - & - & - & - \\
\hline $\begin{array}{l}\text { Klebsiella pneumonia } \\
\quad \text { (ATCC 35657) }\end{array}$ & 4 & 10 & 4 & 10 & 10 & 16 & - & 10 & 10 & 10 & 10 & 10 \\
\hline $\begin{array}{c}\text { Bacillus subtilis } \\
\text { (NCTC 8236) }\end{array}$ & 6 & - & 8 & 20 & 20 & 20 & - & 10 & 10 & 10 & 10 & 10 \\
\hline $\begin{array}{l}\text { Escherichia coli } \\
\text { (ATCC 25922) }\end{array}$ & - & - & 6 & - & - & 14 & 16 & 18 & 12 & 14 & 16 & 14 \\
\hline $\begin{array}{c}\text { Staphylococcus aureus } \\
\text { (ATCC 25923) }\end{array}$ & - & - & - & - & - & - & - & - & - & - & - & - \\
\hline
\end{tabular}


teus mirabilis and $P$. aeruginosa species and the aqueous extract showed the lowest activity. The most polar solvent results in a greater yield extract of natural antioxidant compounds because most of them are polar compounds such as flavonoids. Solvent with higher polarity are effective for extraction of natural antioxidants [32]. The antibacterial activity of $T$. chebula, does not come as a surprise, since sesquiteprene lactones, flavonoids and essential oils in general have been associated several times with antibacterial effect [33] [34].

The aqueous extracts of all plants except $T$. chebula do not possess significant antibacterial activity both against standard and clinical strains. These results account for why the Sudanese people do not frequently use these plants as a remedy, but may possess anti-inflammatory activities.

T. chebula can serve as a starting point in future drug development aimed at the production of a new safe, effective and bio-accessible therapeutic agent. Plants were able to develop new, faster and natural antimicrobials and then man-made remedies, and that is explaining why plants succeed in its fighting against microbes since millions of years while human failed [35].

Methanolic extracts of Commiphora myrrha gumshowed potent antibacterial activity against clinical isolates $P$. mirabilis isolate no. (3) and E. coli isolate no. (6). Furanosesquiterpenes, the active compounds in C. myrrh essential oil, possessed a significant antiseptic property, also be the characteristic components of pharmaceutical myrrh [26] [36]. All standard bacteria showed resistant to methanolic and aqueous extracts. Most of standard bacteria showed antibiotic-resistant. The reason for different sensitivity between Gram-positive and Gram negative bacteria could be ascribed to the morphological differences between these microorganisms. Gram-negative bacteria have an outer phospholipids membrane carrying the structural lipopolysaccharide components. This makes the cell wall impermeable to lipophilic solutes, while porins constitute a selective barrier to the hydrophilic solutes. The Gram-positive bacteria should be more susceptible since they have only an outer peptidoglycan layer which is not an effective permeability barrier [37].

Antibiotic-resistant S. aureus (ATCC 25923) showed promising activity against S. argel leaves. Staphylococcal disease has been a perennial problem in the hospital environment since the beginning of the antibiotic era, hospital strains of $S$. aureus were usually resistant to a variety of different antibiotics. A few strains were resistant to all clinically useful antibiotics except vancomycin, and vancomycin-resistant strains were increasingly reported [38].

Methanolic extracts of $T$. chebula fruits showed high antibacterial activity against $E$. coli and S. aureus which showed antibiotic-resistant to most antibiotic and similarly to amikacin, and S. aureus (12) showed antibioticresistant to most antibiotic and similarly to gatifloxacin. These plants could serve as useful sources for new antimicrobial agents and the fruits extracts of $T$. chebula may be used as remedy against various diseases without any side effects [39].

Staphylococcus has changed from the status of a non-pathogen to that of an opportunistic pathogen. Although once regarded as an important opportunistic innocuous member of the normal skin flora, Staphylococcus now recognized as an important opportunistic pathogen. It is routinely found on the skin and in the hospital environment, with prevalence on the skin surface of $85 \%-100 \%$. Staphylococcus was found excessively on damaged skin surface in normal persons [40].

Of the all S. aureus isolates, only one was more sensitive to themethanolic extract of $T$. chebula fruits and effective more than Gram negative antibiotics, also T. chebula showed high activity against standard $S$. aureus (ATCC 25923) were it showed antibiotic-resistant (Table 1). Study of the synergistic interaction of active phyto-compounds with antibiotics is required to exploit these potential plant extracts in the combination therapy of infectious diseases caused by multi drug-resistant organisms [41].

75\% of $P$. aeruginosa showed high sensitivity toward methanolic extract, Escherichia coli which showed antibiotic-resistant to most antibiotic and similarly to amikacin. In the last decades, prevalence and outbreaks of the multi-drug resistant bacterial strains has been increasingly documented throughout the world. At present most clinical isolates of E. coli areconsidered as highly resistant to most commercially known antibiotics [35]. Out of the 20 clinical isolates from the infected stool, 2 were S. typhi, 5 P. mirabilis, 4 E. coli, 5 P. aeruginosa, $3 S$. aureus, one was $S$ typhi $B$. This is in line with fact that $P$. mirabilis, E. coli and $P$. aeruginosa are the most commonly taxa encountered contaminants of stool in foods. All bacterial species were found to be resistant against aqueous extracts of all studied plant species (except $T$. chebula). Compared to the most reference antibiotics, the spectrum of antibacterial activity of T.chebula was found to be clearly superior (Table $4 \&$ Table 5).

The phytochemistry of Terminalia chebula, Solenostemma argel, Commiphora myrrha, Rutagraveolens and Cistanche phelypaea have been received considerable interest [42]-[46]. Hexane and dichloromethane extracts 
Table 4. Minimum inhibition zone (MIC) and antibacterial activity of crude methanolic extractives—clinical isolates and STD.

\begin{tabular}{|c|c|c|c|c|c|c|c|}
\hline \multirow{2}{*}{ Plants } & \multirow{2}{*}{ Clinical Isolates } & \multicolumn{6}{|c|}{ Concentrations } \\
\hline & & $0.5 \mathrm{mg}$ & $0.3 \mathrm{mg}$ & $0.15 \mathrm{mg}$ & $0.05 \mathrm{mg}$ & $0.03 \mathrm{mg}$ & $0.01 \mathrm{mg}$ \\
\hline \multirow{8}{*}{ Terminalia chebula } & Proteus mirabilis 3 & 20 & 16 & 20 & 18 & 4 & 4 \\
\hline & Proteus mirabilis 4 & 28 & 10 & 10 & 10 & - & - \\
\hline & Escherichia coli 5 & 24 & 4 & 2 & 2 & - & - \\
\hline & Escherichia coli 10 & 28 & 4 & 4 & 2 & - & - \\
\hline & Pseudomonas aeruginosa 13 & 20 & 14 & 14 & 14 & 4 & 2 \\
\hline & Pseudomonas aeruginosa 7 & 20 & 14 & 14 & 12 & 4 & 2 \\
\hline & Pseudomonas aeruginosa 8 & 20 & - & - & - & - & - \\
\hline & Staphylococcus aureus 12 & 20 & 6 & 6 & 2 & - & - \\
\hline \multirow{2}{*}{ Commiphora myrrha } & Proteus mirabilis 3 & 20 & 4 & 4 & 4 & - & - \\
\hline & Escherichia coli 6 & 20 & 6 & 4 & 2 & - & - \\
\hline \multicolumn{8}{|l|}{ Standard bacteria } \\
\hline \multirow{3}{*}{ Terminalia chebul } & Bacillus subtilis (NCTC 8236) & 18 & 12 & 8 & 8 & - & - \\
\hline & Escherichia coli (ATCC 25922) & 6 & 2 & - & - & - & - \\
\hline & Staphylococcus aureus (ATCC 25923) & 24 & 4 & 4 & 4 & - & - \\
\hline Soleno stemma argelarge & Staphylococcus aureus (ATCC 25923) & 24 & 4 & 4 & 4 & - & - \\
\hline
\end{tabular}

Values are the mean of four replicates. Tested concentration of extracts: $100 \mathrm{mg} / \mathrm{ml}(0.1 \mathrm{ml} /$ well).methanol did not show any inhibitory activity. Sensitive: >18, intermediate: 14 - 18 mm, Resistant: <14 mm, -: No inhibition zone; *Standard bacteria; S.a: Staphylococcus aureus (ATCC 25923) B.s: Bacillus subtilis (NCTC 8236), E.c: Escherichia coli (ATCC 25922), K.n: Klebsiella pneumoniae (ATCC 35657), Sa.t: Salmonella typhi (ATCC1319106); Sensitive: >18, intermediate: 14 - 18 mm, Resistant: <14 mm, -: No inhibition zone; ATCC: American Type Collection Culture; NCTC: National Collection Type Culture.

Table 5. Minimum Inhibition zone (MIC) and antibacterial activity of crude aqueous extractives—clinical isolates and STD.

\begin{tabular}{|c|c|c|c|c|}
\hline \multirow{2}{*}{ Plants } & \multirow{2}{*}{ Clinical Isolates } & \multicolumn{3}{|c|}{ Concentrations } \\
\hline & & $100 \%$ & $50 \%$ & $25 \%$ \\
\hline \multirow{5}{*}{ Terminalia chebula } & Salmonella typhi.No 1 & 14 & - & - \\
\hline & Escherichia coli .No 6 & 14 & 10 & 8 \\
\hline & Pseudomonas aeruginosa. No 8 & 14 & 14 & 12 \\
\hline & Salmonella paratyphi B. No 17 & 16 & 8 & 4 \\
\hline & Proteus mirabilis. No 19 & 14 & - & - \\
\hline \multicolumn{5}{|l|}{ Standard bacteria } \\
\hline \multirow{4}{*}{ Terminalia chebula } & Bacillus subtilis (NCTC 8236) & 16 & 20 & 10 \\
\hline & Salmonella typhi (ATCC1319106) & 10 & & \\
\hline & Klebsiella pneumonia (ATCC 35657) & 10 & - & - \\
\hline & Staphylococcus aureus (ATCC 25923) & 14 & 6 & 2 \\
\hline
\end{tabular}

Values are the mean of four replicates. Tested concentration of extracts: $100 \mathrm{mg} / \mathrm{ml}(0.1 \mathrm{ml} /$ well $)$.methanol did not show any inhibitory activity. Sensitive: >18, intermediate: 14 - 18 mm, Resistant: <14 mm, -: No inhibition zone; 'Standard bacteria; S.a: Staphylococcus aureus (ATCC 25923) B.s: Bacillus subtilis (NCTC 8236), E.c: Escherichia coli (ATCC 25922), K.n: Klebsiella pneumoniae (ATCC 35657), Sa.t: Salmonella typhi (ATCC1319106); Sensitive: >18, intermediate: 14 - 18 mm, Resistant: <14 mm, -: No inhibition zone; ATCC: American Type Collection Culture; NCTC: National Collection Type Culture. 
of Terminalia chebula have shown more antibacterial compounds than acetone extract indicating the non-polar character of the antibacterial compounds [42]. Nine flavonoidal compounds were extracted from stem aqueous extract of Solenostemma argel. The kaempferol was more effective as antibacterial agent [43]. Three compounds known for their antibacterial effects of Commiphora myrrha: 2-fluorodiphenylmethane, tribenzo-1, 2,3,4,5,6anthracene and 2-bromo-1-(4-bromophenyl)ethanone [44]. Orlanda and Nascimento mention that $R u$ tagraveolens could be used as a natural source for antibacterial compounds and possible applications in the pharmaceutical industry [45]. Several phenylethanoid glycosides isolated from Cistanche spp. showed an antibacterial activity against Staphylococcus aureus [46]. Therefore, the results obtained in our research work match the results obtained by other researches.

\section{Conclusions}

The most antibacterial active plant was T. chebula fruits. Of all extracts the methanolic and aqueous extracts of T. chebula fruits was the most active, whereas, the aqueous extracts of all plants do not possess significant antibacterial activity both against standard and clinical strains.

Methanol extracts of $C$. myrrha gumand $T$. chebula fruits showed high antibacterial activity against $P$. mirabilis, E. coli, P. aeruginosa, S. aureus, S. typhi and S. typhi B clinical isolates, whereas the aqueous extracts of all plants were found to be ineffective against all tested bacteria Gram-positive and Gram-negative T. chebula fruitswhich showed moderate effect against P. mirabilis, E. coli, P. aeruginosa S. typhi and S. typhi B.

Some standard bacterial species showed a fairly high degree of sensitivity to the methanolic extracts of $T$. chebula fruits against B. subtilis (NCTC 8236) and S. aureus (ATCC 25923), whereas the aqueous extracts of all plants were found to be ineffective against all tested bacteria except $T$. chebula fruits showed moderate potency against $B$. subtilis (NCTC 8236).

The antibacterial screening of the different extracts (methanol and aqueous) was performed against standard and clinically isolated bacterial strains. The highest antibacterial activity was found in methanolic extracts, the lowest one was found in aqueous extract.

\section{References}

[1] Nascimento, G.F., Locatelli, J., Freitas, P.C. and Silva, G.L. (2000) Antibacterial Activity of Plant Extracts and Phytochemicals on Antibiotic-Resistant Bacteria. Brazilian Journal of Microbiology, 31, 1-4. http://dx.doi.org/10.1590/S1517-83822000000400003

[2] Maji, S., Dandapat, P., Ojha, D., Maity, C., Halder,S.K., Das Mohapatra, P.K., Pathak, T.K., Pati, B.R., Samanta, A. and Mondal, K.C. (2010) In Vitro Antimicrobial Potentialities of Different Solvent Extracts of Ethnomedical Plants against Clinically Isolated Human Pathogens. Journal of Phytology, 2, 57-64.

[3] Ahmad, I., Mehmood, Z., Mohammed, F. and Ahmad, S. (2000) Antimicrobial Potency and Synergistic Activity of Five Traditionally used Indian Medicinal Plants. Journal of Medicinal and Aromatic Plant Sciences, 22, 173-176.

[4] Agrawal, A., Gupta, A., Choudhary, N.K., Wadhwa, S., Dave, K., Goyal, S. and Rana, S.S. (2010) Antibacterial Activity of Hydroalcoholic Extract of Terminalia chebula Retz on Different Gram-positive and Gram-negative Bacteria. International Journal of Pharmaceutical and Biological Archives, 1, 485-488.

[5] Bag, A., Bhattacharyya, S.K., Bharati, P., Pal, N.K. and Chattopadhyay, R.R. (2009) Evaluation of Antibacterial Properties of Chebulicmyrobalan (Fruit of Terminalia chebula Retz.)Extracts against Methicillin Resistant Staphylococcus aureusand Trimethoprim-Sulphamethoxazole Resistant UropathogenicEscherichia coli. African Journal of Plant Science, 3, 025-029.

[6] Shahidi, G.H., Nik, A.K., Heydari, M.R., Ghasemzadeh, M.H., Farrokhi, P.R., Moein, M.R., Mansouri, SH. and Foroumadi, A. (2003) Anti-pseudomona and Anti-bacilli Activity of Some Medicinal Plants of Iran. Daru, 11, 157-163.

[7] Naqvi, S.H., Asif, M., Rehman, A.B. and Ahmad, M. (2010) Evaluation of Antimicrobial Properties of Terminalia chebula Retz. Pakistan Journal of Pharmacology, 27, 29-35.

[8] Aneja, K.R. and Joshi, R. (2009) Evaluation of Antimicrobial Properties of Fruit Extracts of Terminalia chebulaagainst Dental Caries Pathogens. Jundishapur Journal of Microbiology, 2, 105-111.

[9] Rahimi, R., Shams-Ardekani, M.R. and Abdollahi, M. (2010) A Review of the Efficacy of Traditional Iranian Medicine for Inflammatory Bowel Disease. World Journal of Gastroenterology, 16, 4504-4514. http://dx.doi.org/10.3748/wjg.v16.i36.4504

[10] Tambekar, D.H., Khante, B.S., Dahikar, S.B. and Zarey, V.M. (2007) Antibacterial Properties of Contents of Triphala: A Traditional Indian Herbal Preparation. Continental Journal of Microbiology, 1, 8-12. 
[11] Sumathi, P. and Parvathi, A. (2010) Antimicrobial Activity of Some Traditional Medicinal Plants. Journal of Medicinal Plants Research, 4, 316-321.

[12] Sharma, A., Verma, R. and Ramteke, P. (2009) Antibacterial Activity of Some Medicinal Plants Used by Tribals against Urinary Tract Infection Causing Pathogens. World Applied Sciences Journal, 7, 332-339.

[13] Panthi, M.P. and Chaudhary, R.P. (2006) Antibacterial Activity of Some Selected Folklore Medicinal Plants from West Nepal. Scientific World, 4, 16-21.

[14] Parekh, J. and Chanda, S. (2006) Screening of Aqueous and Alcoholic Extracts of Some Indian Medicinal Plants for Antibacterial Activity. Indian Journal of Pharmaceutical Sciences, 68, 835-838. http://dx.doi.org/10.4103/0250-474X.31032

[15] Ponjar, G.H., Aghighi, S. and Nik, A.K. (2004) Antibacterial and Antifungal in Plants Used in Indigenous HerbalMedicine of South East Regions of Iran. Journal of Biological Science, 4, 405-412. http://dx.doi.org/10.3923/jbs.2004.405.412

[16] Lee, D., Boo, K.H., Woo, J-K., Duan, F., Lee, K.H., Kwon, T.K., Lee, H.Y., Riu, K.Z. and Lee, D.S. (2011) Anti-bacterial and Anti-viral Activities of Extracts from Terminalia chebula Barks. Journal of Korean Society. Applied Biological Chemistry, 54, 295-298.

[17] Khan, K.H. and Jain, S.K. (2009) Regular Intake of Terminalia chebula Can Reduce the Risk of Getting Typhoid Fever. Advanced Biotech. Advanced Biotech, 8. www.advancedbiotech.net/archives/2.html

[18] Tambekar, D.H., Khante, B.S., Dahikar, S.B. and Zarey, V.M. (2007) Antibacterial Properties of Contents of Triphala: A Traditional Indian Herbal Preparation. Continental Journal of Microbiology, 1, 8-12.

[19] Rahmatullah, M., Islam, T., Hasan, E., Ahmed, R., Jamal, F., Jahan, R., Khatun, A., Nahar, N., Ahsan, S., Nahar, A. and Ahmad, I. (2010) A Survey of Medicinal Plants Used by the Folk Medicinal Practitioners of Shetabganj Village in Dinajpur District, Bangladesh. American-Eurasian Journal of Sustainable Agriculture, 4, 196-203.

[20] Borah, P.K., Gogoi, P., Phukan, A.C. and Mahanta, J. (2006) Traditional Medicine in Treatment of Gastrointestinal Diseases in Upper Assam. Indian Journal of Traditional Knowledge, 5, 510-512.

[21] Sulieman, A.E., Elzobair, W.M. and Abdelrahim, A.M. (2009) Antimicrobial Activity of the Extract of Solenostemma argel (Harjal) Plant. Journal of Science and Technology, 10, 120-134.

[22] Abd Elhady, F.K. (1994) Studies for Determining Antimicrobial Activity of Solenostemma argel (Del) Hayne. 1-Extraction with Methanol/Water in Different Proportions. Qatar University Science Journal, 14, 138-142. http://hdl.handle.net/10576/10261

[23] Benhouhou, S., Batanouny, K, AbdelRahman, F., et al. (2005) A Guide of Medicinal Plants in North Africa. IUCN. https://portals.iucn.org/library/sites/library/files/...../2005-093.pdf

[24] Wanner, J., Schmidt, E., Bail, S., Jirovetz, L., Buchbauer, G., Gochev, V., Girova, T., Atanasova, T. and Stoyanova, A. (2010) Chemical Composition and Antibacterial Activity of Selected Essential Oils and Some of Their Main Compounds. Natural Product Communications, 5, 1359-1364.

[25] El-Kamali, H.H. (2009) Medicinal Plants in East and Central Africa: Challenges and Constraints. Ethnobotanical Leaflets, 13, 364-369.

[26] Lemenih, M. and Teketa, D. (2003) Frankincense and Myrrh Resources of Ethiopia: II. Medicinal and Industrial Uses. SINET: Ethiopian Journal of Science, 26, 161-172.

[27] Grace, D. (2011) Myrrh Gum.Malaria.ws. A Botanical Search for a Cure Proudly Powered by Word Press. http://malaria.ws/wp-content/uploads/2011/06/myrrh.jpg

[28] Koua, F.H., Babiker, H.A., Halfawi, A., Ibrahim, R.O., Abbas, F.M., Elgaali, E.I. and Khlafallah, M.M. (2011) Phytochemical and Biological Study of Striga hermonthica (Del.) Benth Callus and Intact Plant. Pharmaceutical Biotechnology, 3, 85-92.

[29] Cruikshank, R. (1975) Medical Microbiology: A Guide to Diagnosis and Control of Infection. E and S Livingston Ltd., Edinburgh and London, 888.

[30] Cheesbrough, M. (1984) Culture Media. In: Cheesbrough, M., Ed., Medical Laboratory Manual for Tropical Countries, Vol. 3, Tropical Health Technology and Butterworth-Heineman, Cambridge, 60-69, 407-428.

[31] Ojala, T., Remes, S., Haansuu, P., Vuorela, H., Hiltunen, R., Haahtela, K. and Vuorela, P. (2000) Antimicrobial Activity of Some Coumarin Containing Herbal Plants Growing in Finland. Journal of Ethnopharmacology, 73, $299-305$. http://dx.doi.org/10.1016/S0378-8741(00)00279-8

[32] Nurul, M.H., Radzali, M., Johari, R., Syahida, A. and Maziah, M. (2008) Antioxidant Activities of Different Aerial Parts of Putat (Barringtonia racemosa L.). Malaysian Journal of Biochemistry and Molecular Biology, 16, 15-19.

[33] Abdel-Mogib, M., Jakupovic, J., Dawidar, A.M., Metwally, M.A. and Abou-Elzahab, M. (1990) Sesquiterpene Lactones and Kaurane Glycosides from Francoeuria crispa. Phytochemistry, 29, 2581-2584. 
http://dx.doi.org/10.1016/0031-9422(90)85193-J

[34] El-kamali, H.H., Ahmed, A.H., Mohammed, A.S., Yahia, A.A.M., Eltayeb, I.H. and Ali, A.A. (1998) Antibacterial Properties of Essential Oil from Nigella sativa Seeds, Cymbopogon citrates Leaves and Pulicaria undulata Aerial Parts. Fitoterapia, 69, 77-78.

[35] Abdallah, E.M. (2011) Plants: An Alternative Source for Antimicrobials. Journal of Applied Pharmaceutical Science, 1, 16-20.

[36] Nomicos, E.Y. (2007) Myrrh: Medical Marvel or Myth of the Magi? Holistic Nursing Practice, 21, 308-323. http://dx.doi.org/10.1097/01.HNP.0000298616.32846.34

[37] Ariasa, M.E., Gomeza, J.D., Cudmanib, N.M., Vattuonec, M.A. and Islac, M.I. (2004) Antibacterial Activity of Ethanolic and Aqueous Extracts of Acacia aroma Gill. ex Hook et Arn. Life Sciences, 75, 191-202. http://dx.doi.org/10.1016/j.lfs.2003.12.007

[38] Todar, K. (2009) Staphylococcus and Staphylococcal Disease. Lectures in Microbiology. http://www.textbookofbacteriology.net

[39] Khan, J.A. and Hanee, S. (2011) Antibacterial Properties of Punica granatum Peels. International Journal of Applied Biology and Pharmaceutical Technology, 2, 23-27.

[40] Subrahmanyam, M., Hemmady, A. and Pawar, S.G. (2001) Antibacterial Activity of Honey on Bacteria from Wounds. Annals of Burns and Fire Disasters, XIV.

[41] Ahmad, I. and Beg, A.Z. (2001) Antimicrobial and Phytochemical Studies on 45 Indian Medicinal Plants against Multi-Drug and Resistant Human Pathogens. Journal of Ethnopharmacology, 74, 113-123. http://dx.doi.org/10.1016/S0378-8741(00)00335-4

[42] Shinde, S.L., Junne, S.B., Wadje, S.S. and Baig, M.M. (2009) The Diversity of Antibacterial Compounds of Terminalia Species (Combretaceae). Pakistan Journal of Biological Sciences, 12, 1483-1486. http://dx.doi.org/10.3923/pjbs.2009.1483.1486

[43] Shafek, R.E., Shafik, N.H. and Michael, H.N. (2012) Antibacterial and Antioxidant Activities of Two New Kaempferol Glycosides Isolated from Solenostemma argel Stem Extract. Asian Journal of Plant Sciences, 11, 143-147. http://dx.doi.org/10.3923/ajps.2012.143.147

[44] Cowan, M.M. (1999) Plants Products as Antimicrobial Agents. Clinical Microbiology Reviews, 12, 564-582.

[45] Oranda, J.F.F. and Nascimento, A.R. (2015) Chemical Composition and Antibacterial Activity of Ruta graveolens L. (Rutaceae) Volatile Oils from Sao Luis, Maranhao, Brazil. South African Journal of Botany, 99, 103-106. http://dx.doi.org/10.1016/j.sajb.2015.03.198

[46] Deyama, T., Kobayashi, H., Nishibe, S. and Tu, P. (2006) Isolation, Structure Elucidation and Bioactivities of Phenylethanoid Glycosides from Cistanche, Forsythia and Plantago Plants. Studies in Natural Products Chemistry, 33, 645-674. http://dx.doi.org/10.1016/S1572-5995(06)80036-0 